\title{
Uptake and loss of tears from filter paper discs employed in lysozyme tests
}

\author{
TERENCE J. HYPHER \\ From the Department of Ophthalmology, University of Liverpool
}

SUMMARY Aspects of the use of filter paper discs in collecting tear samples for lysozyme tests were studied by serial weighings of bijoux bottles and tear-moistened discs. Blotted discs carried from 1.9 to $3.6 \mathrm{mg}$ tears, but for practical purposes no deposit was left in the bottle. Unblotted discs carried a greater quantity of tear fluid, but the majority deposited a proportion of this in the bottle, and a correcting factor is therefore proposed for the lysozyme assay technique of Mackie and Seal. Substantial vaporisation of tears occurred within the bottles.

Filter paper discs may be employed as a means of collecting tear fluid for estimation of lysozyme activity. In the assay of tear lysozyme it is necessary that each tear sample be of constant quantity or that individual weights of samples are known. Van Bijsterveld (1969) collected tear samples by placing a 6-mm filter paper disc in the lower conjunctival fornix, allowing it to become entirely wet, and then blotting off excess fluid between a filter paper. In his results possible variation in tear content of the disc was not considered. Mackie and Seal (1976) collected tear samples in a similar way but without blotting the discs. The quantity of tears collected was determined by weighing a bijou bottle containing the 6-mm filter paper disc first dry and then after the disc had been soaked with tears. The filter paper was then removed from the bottle and transferred to an agar plate for lysozyme estimation. They found that the quantity of tears held by the unblotted disc could vary by up to fourfold, but, with the known weight of tears collected being taken into account, the lysozyme concentration was expressed in units of activity per microlitre of tears. Subsequently the method has also been employed in a slightly modified form, a $5-\mathrm{mm}$ disc being placed in a $30 \times 10 \mathrm{~mm}$ tube (Mackie et al., 1977).

Two questions arise from the use of these techniques: firstly, whether it is correct to assume that blotting the discs will result in a constant weight of tears in each sample; secondly, whether discs, either blotted or unblotted, may leave behind a deposit of

Address for reprints: Mr T. J. Hypher, FRCS, 16 The Crescent, Waterloo Park, Liverpool L22 3XH surplus tears in the bottle in which they are weighed. A trial was undertaken to answer these 2 questions and in addition to examine the possibility of vaporisation of tears within the bottles.

\section{Methods}

Fifty normal volunteers (ages 17 to 59 years) agreed to take part in the trial, and two procedures were followed.

FIRST PROCEDURE

Empty bijoux bottles were weighed. A sterile Whatman No. 1 filter paper disc, $6 \mathrm{~mm}$ in diameter, was placed in the lower conjunctival fornix by means of forceps and allowed to remain until it was seen to be soaked. From 1 eye the disc was removed and placed directly into a bijou bottle, and allowed to rest on the side for 2 to 3 seconds. It was then withdrawn from the bottle and discarded, and the bottle tightly closed. From the second eye exactly the same technique was followed, except that, prior to placing the soaked disc in the bottle, it was allowed to rest on a Whatman No. 1 filter paper for a few seconds until it could be seen that all surplus tears had been absorbed. Within 2 hours, and usually within 1 hour, the bijoux bottles were reweighed to determine whether any tears had been deposited by contact with the discs.

\section{SECOND PROCEDURE}

This was undertaken on a separate occasion from the first procedure. Bijoux bottles were weighed, each containing a sterile 6-mm filter paper disc. The disc was taken from the bottle and placed in the 


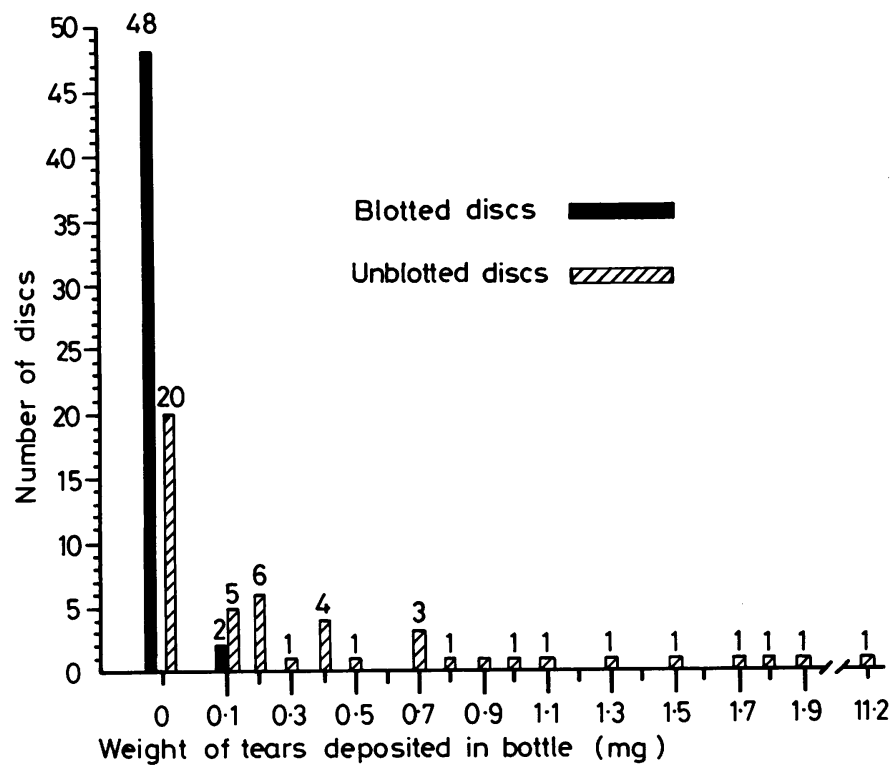

Fig. 1 Tears deposited in bottle by contact with disc

lower fornix and allowed to remain until soaked. From 1 eye this was then returned directly to the bottle, which was immediately closed. From the second eye the disc was blotted, as in the first procedure, and then returned to the bottle. Within 2 hours the bottles were weighed to determine the quantity of tears absorbed by the discs. Each disc was then removed from the bottle and weighed wet. Finally, after it had been allowed to dry out completely at room temperature the disc was again weighed.

\section{Results}

FIRST PROCEDURE

Tears deposited in bottle. Results of the first procedure are displayed in Fig. 1, where it can be seen that of the 50 blotted discs 48 left no measurable tear deposit in the bottle, while the remaining 2 each deposited $0.1 \mathrm{mg}$. Of the 50 unblotted discs 20 left no tear deposit, 29 left measurable weights up to $1.9 \mathrm{mg}$, and 1 disc deposited $11.2 \mathrm{mg}$. (A Kolmogorov-Smirnov 2-sample test (2-tailed) (Siegel, 1956) gave $P<0.001$, showing a highly significant difference between the blotted and unblotted samples.)

SECOND PROCEDURE

Tears absorbed by discs. Fig. 2 shows the blotted discs to hold 1.9 to $3.6 \mathrm{mg}$ tears (mean $2.57 \mathrm{mg}$, SD 0.36). The unblotted discs can be seen to have absorbed from 2.2 to $8.9 \mathrm{mg}$, with one exceptional disc holding $15 \cdot 1 \mathrm{mg}$ tears (mean $5 \cdot 25 \mathrm{mg}$, SD 2.13)
(Kolmogorov-Smirnov 2-sample test (2-tailed) bebetween blotted and unblotted samples: $P<0.001)$.

Tears remaining in discs on removal from bottle. These results are introduced in the discussion of loss of tears by vaporisation.

\section{Discussion}

\section{BLOTTED DISCS}

Fig. 1 shows that only 2 blotted discs deposited a measurable weight of tears on contact with the bijou bottle. In each case this was $0.1 \mathrm{mg}$, the smallest weight detectable by the balance, and its loss would not affect the lysozyme test. For practical purposes, therefore, it may be accepted that no tear deposit occurred from the blotted discs.

The tear weight in the blotted discs (Fig. 2) has been seen to be inconstant and to range from 1.9 to $3.6 \mathrm{mg}$ about the mean $2.57 \mathrm{mg}$ (SD 0.36). In the statistical analysis of his diameters of lysis van Bijsterveld (1969) obtained a variance of 0.2 (representing a change in zone of lysis of $0.45 \mathrm{~mm}=$ $1 \mathrm{SD})$ both within normal persons and between eyes within normal persons. He did not consider the possibility of variation in tear weight in the discs but interpreted the variance as an alteration in the individual. A lysozyme concentration/diameter of lysis curve is not given in van Bijsterveld's paper, which precludes a direct calculation of the variation in zone of lysis which would result from change in tear content in his filter paper discs. (Van Bijsterveld employed Whatman No. 3 filter paper. Per unit 
Fig. 2 Weights of tears absorbed by discs

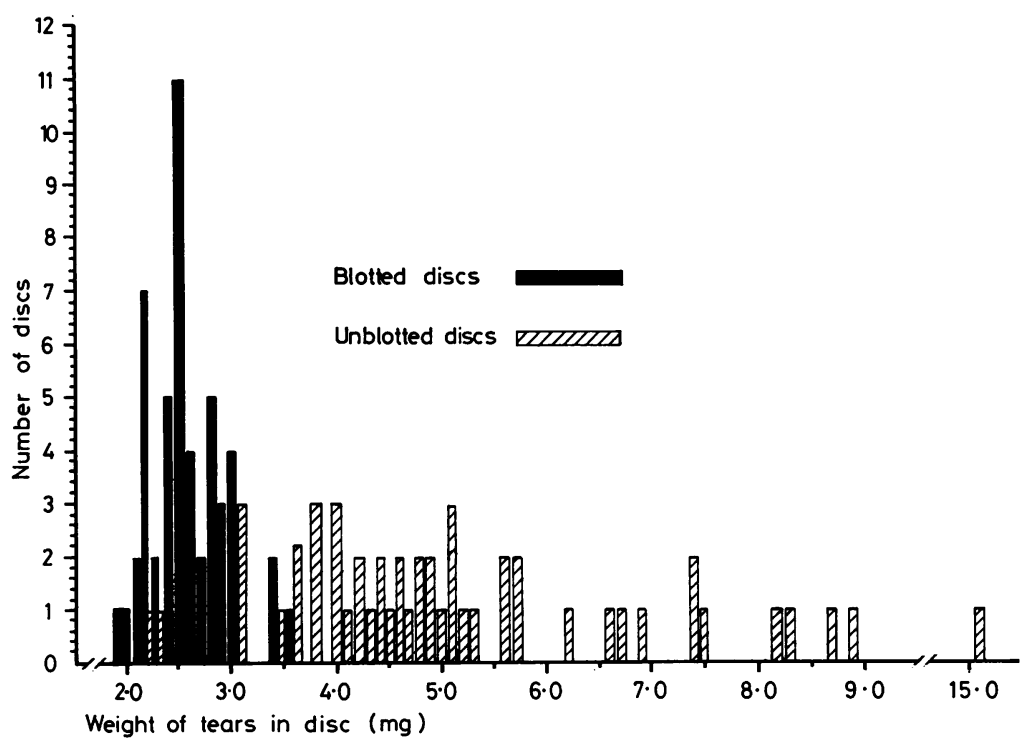

weight of paper this absorbs $7 \%$ more fluid than Whatman No. 1, but the variation in uptake is closely comparable in the 2 papers.)

However, estimation of this variation is possible in the case of published curves. Two examples are presented in Table 1 , giving the alteration in diameter of zone of lysis which would result from $14 \%$ change in tear weight in the disc $(14 \%$ change $\equiv$ $0.36 \mathrm{mg}$ (1 SD) above or below mean tear weight $2.57 \mathrm{mg}$; estimation made on curve at mean lysozyme concentration in normal eyes). It is seen that the magnitude of zone variation is closely comparable with the figure of $0.45 \mathrm{~mm}$ found by van Bijsterveld. It is concluded, therefore, that variances of this degree found by van Bijsterveld could be attributed to alteration in tear volume in the discs rather than a presumed qualitative change in the tears of the individual. These findings do not, however, affect his conclusions on the reliability of his lysozyme test, the variances having already been taken into account in his analysis.

Table 1 Changes in diameter of zone of lysis resulting from increasing and decreasing tear weight by $14 \%$ (equivalent to a change of $0.36 \mathrm{mg}$ above and below mean $2.57 \mathrm{mg}$ )

\begin{tabular}{lll}
\hline Paper & Increase in diameter & Decrease in diameter \\
\hline $\begin{array}{c}\text { Bonavida and Sapse } \\
\text { (1968, Fig. 1) }\end{array}$ & $0.36 \mathrm{~mm}$ & $0.41 \mathrm{~mm}$ \\
$\begin{array}{c}\text { Pietsch and Pearlman } \\
(1973, \text { Fig. 1) }\end{array}$ & $0.55 \mathrm{~mm}$ & $0.63 \mathrm{~mm}$ \\
\hline
\end{tabular}

\section{UNBLOTTED DISCS}

Substantial quantities of tears were deposited in bijoux bottles by many of the unblotted discs (Fig. 1). This deposit would be of whole tears, that is, containing lysozyme, the loss of which would affect the results of a lysozyme test. If it is assumed that the weight of tear deposit occurring from a disc is proportional to the quantity carried, the deposits (Fig. 1) can be related directly to the quantities in the discs (Fig. 2), disc for disc in order of tear weight. In Fig. 3 the fraction of tears lost from each disc by contact with the bottle, estimated in this manner, is plotted against the original weight of tears in the disc. It is seen that for discs carrying up to approximately $4.5 \mathrm{mg}$ tears no loss occurs, but with larger quantities tears are deposited in increasing propotion, there being a remarkably close correlation between the two variables (correlation coefficient $=$ $0.97, \mathrm{P}<0.001$ ).

Had the lysozyme assay technique of Mackie and Seal (1976) been performed on the tear samples in this trial there would have been an underestimation of lysozyme activity ranging from 2 to $22 \%$ in 29 cases, but in the exceptional tear sample of $15.1 \mathrm{mg}$ the error would be likely to have exceeded $60 \%$. In their paper Mackie and Seal (1976) report mean tear volumes collected of $5 \cdot 1,4 \cdot 5$, and $4 \cdot 3 \mu \mathrm{l}$ (largest volume $8.5 \mu \mathrm{l})$. This indicates that approximately $50 \%$ of their samples exceeded $4.5 \mathrm{mg}$ in weight and will have left deposits in the bijoux bottles of up to a maximum $24 \%$ of tear weight. The estimations of lysozyme concentration will therefore be deficient in these cases, and the mean lysozyme 


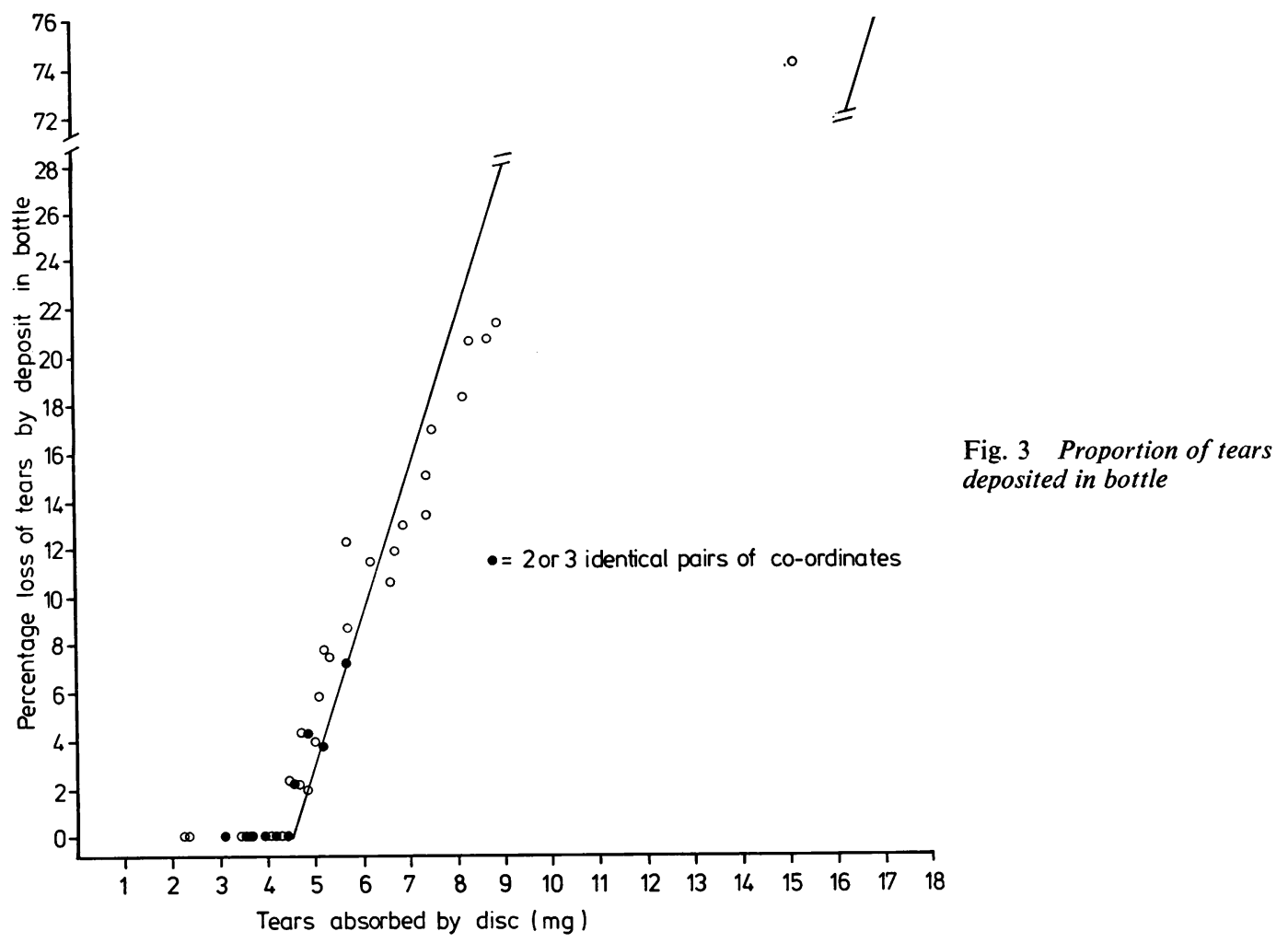

concentration line (Mackie and Seal, 1976, Fig. 3) will be lower than the true mean. It is also possible that the critical level is similarly incorrect. However, without knowledge of the weight of each individual tear sample the exact error cannot be determined.

It is possible to overcome this underestimation by applying a correcting factor to determine the tear weight remaining in the disc after any deposit has occurred in the bottle. For tear weights up to $4.5 \mathrm{mg}$, as measured by weighing the bottle containing the moistened disc, no correction is required. With weights exceeding $4.5 \mathrm{mg}$ :

$$
D=1.27 b-0.061 b^{2}
$$

where $D=$ weight of tears remaining in the disc after deposit has occurred, and $b=$ original tear weight (bottle weighed containing moistened disc).

The above formula relates to 6-mm discs and may not necessarily be correct where glass bottles are replaced by plastic tubes. The alternative is to blot the discs and thus prevent loss of tears by contact with the container.

LOSS OF TEARS BY VAPORISATION

While deposit of tears in the bottle is loss of whole tears, resulting in error in lysozyme estimation, loss by evaporation will not alter the lysozyme content of the disc. It will, however, affect the weighing of tear samples.

The weight of tears remaining in each disc on removal from the bottle was determined from wet and dried disc weights. This figure was subtracted from the original weight of tears collected, the difference representing the weight of tears remaining in the bottle. In the case of the blotted discs this was due to vaporisation within the bottle, there being for practical purposes no contact deposit of whole tears. The tears left in the bottle by the unblotted discs were a combination of tear deposit and vapour, but by subtraction of the estimated deposit the figure for vaporisation was obtained.

It was found that moisture remaining in the bottle from vaporisation was: blotted discs 0 to $32 \%$ of tear weight (mean 14.6\%); unblotted discs 0 to $36 \%$ of tear weight (mean $17 \cdot 2 \%$ ). In neither case was there a significant relationship between original tear weight and loss by vaporisation $(P>0.5)$.

It is clear, therefore, that a substantial proportion of tears may vaporise inside the bottle, and in techniques where tear weight is taken into account it is important that the discs are weighed while 
still enclosed within the bottle, as, for example, in the method of Mackie and Seal (1976).

I am indebted to Dr A. Percival for technical advice, to Dr M. J. Coombs and Dr J. R. Green for statistical advice, and Mr R. Mapstone and Mr S. I. Davidson for support and encouragement.

\section{References}

Bijsterveld, O. P. van (1969). Diagnostic tests in the sicca syndrome. Archives of Ophthalmology, 82, 10-14.

Bonavida, B., and Sapse, A. T. (1968). Human tear lysozyme.
II. Quantitative determination with standard Schirmer strips. American Journal of Ophthalmology, 66, 70-76.

Mackie, I. A., and Seal, D. V. (1976). Quantitative tear lysozyme assay in units of activity per microlitre. British Journal of Ophthalmology, 60, 70-74.

Mackie, I. A., Seal, D. V., and Pescod, J. M. (1977). Betaadrenergic receptor blocking drugs: tear lysozyme and immunological screening for adverse reaction. British Journal of Ophthalmology, 61, 354-359.

Pietsch, R. L., and Pearlman, M. E. (1973). Human tear lysozyme variables. Archives of Ophthalmology, 90, 94-96.

Siegel, S. (1956). Nonparametric Statistics for the Behavioral Sciences, International Student edition, pp. 127-136. McGraw-Hill Kogakusha: Tokyo. 